“C 2006 IEEE. Personal use of this material is permitted. Permission from IEEE must be obtained for all other uses, in any current or future media, including reprinting/republishing this material for advertising or promotional purposes, creating new collective works, for resale or redistribution to servers or lists, or reuse of any copyrighted component of this work in other works." 


\title{
Estimation of Oxygen Consumption for Moderate Exercises by Using a Hammerstein Model
}

\author{
Steven W. Su ${ }^{\dagger a}$, Lu Wang ${ }^{\dagger}$, Branko G. Celler ${ }^{\dagger *}$, and Andrey V. Savkin ${ }^{\dagger}$
}

\begin{abstract}
This paper aims to establish block-structured model to predict oxygen uptake during moderate treadmill exercises. To model the steady state relationship between oxygen uptake (oxygen consumption) and walking speed, six healthy male subjects walked on a motor driven treadmill with constant speed from 2 to $7 \mathrm{~km} / \mathrm{h}$. The averaged oxygen uptake at steady state $\left(\mathrm{VO}_{2}\right)$ was measured by a mixing chamber based gas analysis and ventilation measurement system (AEI Moxus Metabolic Cart). Based on these reliable date, a nonlinear steady state relationship was successfully established by using Support Vector Regression. In order to capture the dynamics of oxygen uptake, a suitable Pseudo Random Binary Signal (PRBS) input was implemented on treadmill for the exercisers. Breath by breath analysis of all subjects was performed. A useful ARX model is identified to justify the measured oxygen uptake dynamics within the aerobic range. Finally, a Hammerstein is achieved, which is useful for the control system design of oxygen uptake regulation during treadmill exercises.
\end{abstract}

Index Terms-Oxygen uptake; Support Vector Regression; Hammerstein model; identification; treadmill exercise; PRBS.

\section{INTRODUCTION}

$\mathbf{O}$ XYGEN uptake is an important physiological parameter for the determination of functional health status and clinical assessments in normal and pathological conditions. The main goal of this paper is to establish practical models to dynamically estimate oxygen uptake for walking exercisers. These models are potentially applicable for the regulation of oxygen uptake during treadmill exercise.

Papers about oxygen uptake modeling for moderate exercise can be divided into two categories: oxygen uptake estimation for the steady state condition (oxygen consumption $\left.\mathrm{VO}_{2}\right)$ and dynamic response characterization during onset and offset exercises. For the prediction of steady state oxygen uptake, book [1] proposed a linear static model to approximately estimate oxygen consumption in a given walking speed ranges. Papers [2] [3] [4] provided simple static nonlinear (polynomial) models.

The oxygen uptake kinetic description at the onset and offset of leg work was first reported by Hill and Lupton [5] in 1923. After that, many papers attempt to capture both the exact time course of these kinetic adjustments and the physiological events governing the rate of adjustment. Most of them [6] [7] approximate the process by using first

\footnotetext{
${ }^{\dagger}$ Human Performance Group, Biomedical Systems Lab, School of Electrical Engineering \& Telecommunications University of New South Wales,UNSW Sydney N.S.W. 2052 Australia.

${ }^{a}$ Faculty of Engineering, University of Technology, Sydney, Australia.

*Author to whom correspondence should be addressed. Email: b.celler@unsw.edu.au. Tel: +61 (2) 9385-4009. Fax: +61 (2) 9385-6743.

This research was supported by the Australian Research Council (Grant DP0452186).
}

order linear models, which were often obtained by using step change responses. Papers [8] [9] applied PRBS and sine signals to bicycle exercises. By using spectrum analysis, they proved the dynamic linearity of oxygen uptake with work load at low frequency.

In this paper, the steady state and dynamics of oxygen uptake during treadmill walking will be capture by using only one Hammerstein model. A novel model identification method for Hammerstein model will also be presented. The Hammerstein model can be described as a static nonlinear block followed by a dynamic linear system. Hammerstein models may account for nonlinear effects encountered in not only industrial processes [10] [11], but also physiological processes [12] [13]. For example, the electrically stimulated muscle [14] and lung tissue strip mechanics [15] can be effectively modeled by Hammerstein models.

The reason for using a Hammerstein model to predict oxygen uptake from treadmill speed is based on the following facts. The static relationship from treadmill speed to work load is not necessarily linear. The dynamic linearity from work load to oxygen uptake was proved by [8]. Therefore, it is reasonable to describe the relationship from treadmill speed to oxygen uptake by a nonlinear static element cascaded by a linear dynamic element.

The modeling of Hammerstein model is a hot research topic. Recently, paper [16] presented a novel overparametrization (two-stage procedures [17]) identification approach for Hammerstein systems. The most distinguish part of that approach is the utility of a powerful machine learning method, Least Square Support Vector Machine (LSSVM). This novel machine learning method sets that paper apart from existing papers.

Support Vector Machine based regression [18] (Support Vector Regression (SVR)) is a new technique, which has been successfully applied to nonlinear function estimation. This paper applies SVM approaches combining with stochastic method [19] to identify physiological processes. There are at least two aspects which are different with paper [16]:

Instead of over parameterization method [20], the stochastic method [19] is employed. As discussed in [19] and [21], the error of the identification of Hammerstein model is not only from linear and static nonlinear part themselves but also from the coupling between them. The pseudo-random binary sequences (PRBS) are applied to decouple the identification of the two parts as suggested in [19]. Another main difference of this approach with [16] is the usage of $\epsilon$-insensitivity SVM [22] [23] instead of LS-SVM [24] [25] [26]. Both LS-SVM and $\epsilon$-insensitivity SVM have the merits of SVM approaches. 
However, the loss function used by $\epsilon$-insensitivity SVM, only penalizes errors greater than a threshold $\epsilon$. This leads to a sparse representation of the decision rule giving significant algorithmic and representation advantages [22]. On the other hand, the ridge regression ( $\epsilon=0)$ used by LS-SVM typically causes the loss of sparseness representation.

It should be emphasized that the proposed Hammerstein model identification approach is especially suitable for the identification of oxygen uptake model. For steady state oxygen uptake measurement, the most suitable way is to employ a mixing chamber to interrupt gas flow and thus prevent streaming of gases and uneven gas concentrations. However, gas samples collected from a mixing chamber are averaged gas fractions over time. The sensitivity to changes in oxygen uptake is therefore reduced [27]. In order to capture dynamical characteristics, breath by breath analysis should be applied for dynamical experiments. However, this may lead to the degrading of measurement accuracy in steady state test. Due to the totally decoupling of static part identification (based on steady state experiment data) and dynamic part identification (based on dynamic experiment data), it is permitted to apply different measurement approaches for steady state tests and dynamic experiments respectively. Therefore, a better estimation result is attainable.

The paper is organized as follows. The details of SVM based Hammerstein model identification approach is given in Section 2. Section 3 presents the application of the proposed approach for the modeling of oxygen uptake during treadmill exercises.

\section{PRoposed SVM BASED HAMMERSTEIN MODEL IDENTIFICATION APPROACH}

In [19], Bai showed that the identification of linear part of a Hammerstein model can be decoupled from nonlinear part with the help of the PRBS input. The reason is any static nonlinearity can be exactly characterized by a linear function under PRBS input which has the binary nature. In this study, the PRBS input is also employed. Thus, the identification of Hammerstein model can be obtained by the identification of static nonlinearity and linear dynamic separately.

As suggested in [19], the steady state gain of the linear dynamic model is constrained to be unity. The steady state characteristic of the Hammerstein system is considered by the static nonlinearity. It should be mentioned that for the identification of the static nonlinearity part, steady state experiments should be made because the PRBS inputs often do not excite the nonlinearity sufficiently in our interested range. Now, we introduce the so called $\epsilon$-insensitivity SVR based static nonlinearity modeling first [28].

Let $\left\{u_{i}, y_{i}\right\}_{i=1}^{N} \subseteq \mathcal{R}^{d} \times \mathcal{R}$ be the inputs and outputs of the data of a Hammerstein system measured in steady state. The goal of the support vector regression is to find a function $f(u)$ which has the following form

$$
f(u)=w \cdot \phi(u)+b,
$$

where $\phi(u)$ represents the high-dimensional feature spaces which are nonlinearly transformed from $u$. The coefficients $w$ and $b$ are estimated by minimizing the regularized risk function:

$$
\frac{1}{2}\|w\|^{2}+C \frac{1}{N} \sum_{i=1}^{N} L_{\epsilon}\left(y_{i}, f\left(u_{i}\right)\right)
$$

The first term is called the regularized term. The second term is the empirical error measured by $\epsilon$-insensitivity loss function which is defined as:

$$
L_{\epsilon}\left(y_{i}, f\left(u_{i}\right)\right)=\left\{\begin{array}{cl}
\left|y_{i}-f\left(u_{i}\right)\right|-\epsilon, & \left|y_{i}-f\left(u_{i}\right)\right|>\epsilon \\
0, & \left|y_{i}-f\left(u_{i}\right)\right| \leq \epsilon
\end{array}\right.
$$

This defines a $\epsilon$ tube. The radius $\epsilon$ of the tube and the regularization constant $C$ are both determined by user.

By solving this constrained optimization problem, we have

$$
f(u)=\sum_{i=1}^{N} \beta_{i} \phi\left(u_{i}\right) \cdot \phi(u)+b .
$$

As mentioned before, by the use of kernels, all necessary computations can be performed directly in input space, without having to compute the map $\phi(u)$ explicitly. After introducing kernel function $k\left(u_{i}, u_{j}\right)$, the above equation can be rewritten as follows.

$$
f(u)=\sum_{i=1}^{N} \beta_{i} k\left(u_{i}, u\right)+b
$$

Where the coefficients $\beta_{i}$ corresponding to each $\left(u_{i}, y_{i}\right)$, and only the so-called support vectors can have nonzero coefficients.

For linear support regression, the kernel function is thus the inner product in the input space:

$$
f(u)=\sum_{i=1}^{N} \beta_{i}<u_{i}, u>+b .
$$

For nonlinear SVR, there are a number of kernel functions which have been found to provide good generalization capabilities, such as polynomials, Radial basis function (RBF), sigmod. Here we give the polynomials and RBF kernel functions as follows:

RBF kernel: $k\left(u, u^{\prime}\right)=\exp \left(-\frac{\left\|u-u^{\prime}\right\|^{2}}{2 \sigma^{2}}\right)$,

Polynomial kernel: $k\left(u, u^{\prime}\right)=\left(\left(u \cdot u^{\prime}\right)+b\right)^{d}$.

Detailed discussion about SVR, such as the selection of radius $\epsilon$ of the tube, kernel function, and the regularization constant $C$, can be found in [29] [28].

As PRBS input is employed to the identification of Hammerstein system, the identification of linear part can be totally decoupled from the nonlinear part. As shown in equation (2.3) of [19], the identification of a Hammerstein model can be simplified as a linear identification problem. Any linear identification approach can be applied. Here, the parametric approach as suggested in [19] is adopted.

\section{MODELING OF OXYGEN UPTAKE BY USING A HAMMERSTEIN MODEL}

In this section, a Hammerstein model will be set up to estimate oxygen uptake from walking speed by using the introduced approach. 


\section{A. Experiment equipments}

The computer controlled treadmill and its related data collection and processing system are shown in Fig. 1.

The treadmill used in the system is the Powerjog " $G$ " Series fully motorized medical grade treadmill manufactured by Sport Engineering Limited, England. Control of the treadmill can be achieved through an RS232 serial port. The treadmill can receive commands from the computer controller via this link, and obeys such commands without supervision. In order to implement PRBS type signal on treadmill, a computer based control system is implemented which can control the speed of treadmill with satisfactory dynamics. During experiments, all signals are synchronized with the PRBS signal. The synchronization of signals was monitored by triaxial accelerometers.

The measurement of oxygen uptake (either averaged or breath by breath recorded) is implemented by using AEI Moxus Metabolic Cart.

\section{B. Nonlinear component modeling by using Support Vector Regression}

In order to identify the nonlinear relationship, steady state experiments are performed. Six young healthy male subjects volunteered to participate in the study. Their physical characteristics are presented in Table I.

\begin{tabular}{cccc}
\hline & Mean & SD & Range \\
\hline Age $(\mathrm{yr})$ & 31.61 & 5.78 & $23-37$ \\
\hline Height $(\mathrm{cm})$ & 176.41 & 5.48 & $169-184$ \\
\hline Body mass $(\mathrm{kg})$ & 74.31 & 9.35 & $60-85$ \\
\hline
\end{tabular}

TABLE I

SUBJECT CHARACTERISTICS $(\mathrm{N}=6)$

All experiments were conducted in the afternoon, and the subjects were permitted to have a light meal one hour before measurements were recorded. Initially, the subjects were asked to walk for about 10 minutes on the treadmill to familiarize them with the experiment. The subjects were then requested to walk at five levels of different speeds (3, 4, 5,6 and $7 \mathrm{~km} / \mathrm{h}$ ). Each level took a total period of 5 minutes, and was followed by a 10-minute resting period. The oxygen uptake was recorded and averaged in every two minutes by using a mixing chamber based gas analysis and ventilation measurement system (AEI Moxus Metabolic Cart). Finally, in order to identify linear dynamic part of the Hammerstein system, subjects were also requested to walk on the treadmill under a PRBS input. Throughout the experiments, the breath

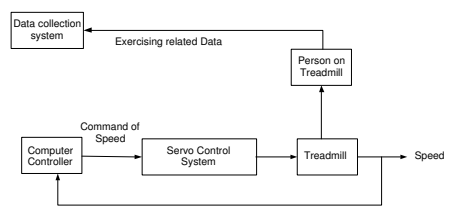

Fig. 1. Block diagram for experimental settings

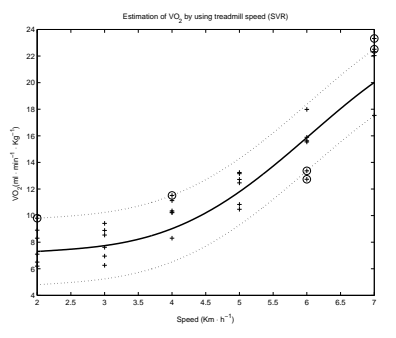

Fig. 2. The steady state relationship captured by using SVR

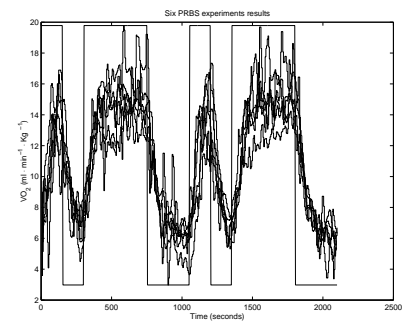

Fig. 3. The oxygen uptake of all six subjects under a PRBS input

by breath tidal volume and the concentration of oxygen were recorded to calculate breath by breath oxygen uptake. The outputs of triaxial accelerometers were also recorded.

In this paper, both traditional linear regression and the $\epsilon$ insensitivity SVR regression method are applied to modeling the nonlinear part. The regression error (Root Mean Square error) of SVR (1.66) is much smaller than linear regression error (2.17). The SVR regression results are summarized in Table II and Fig. 2.

\begin{tabular}{ccc}
\hline Kernel & Parameter & Regularization constant C \\
\hline RBF & $\sigma=3$ & 50 \\
\hline \hline$\epsilon$-insensitivity & Support vectors number & RMS error \\
\hline 2.5 & 6 & 1.66 \\
$\left(\mathrm{ml} \cdot \mathrm{min}^{-1} \cdot \mathrm{Kg}^{-1}\right)$ & $(17.1 \%)$ & $\left(\mathrm{ml} \cdot \mathrm{min}^{-1} \cdot \mathrm{Kg}^{-1}\right)$ \\
\hline
\end{tabular}

TABLE II

DETAILS ABOUT THE ESTIMATION OF THE NONLINEARITY BY SVR

\section{Modeling for linear dynamic part}

The oxygen uptake of all six subjects under a PRBS input is shown in Fig. 3.

The averaged oxygen uptake of six experiments is shown in Fig. 4. Papers [5] [30] [31] often select first order exponential, with no time delays to describe the dynamics of oxygen uptake. In this study, we also adopt first order exponential to describe the dynamics. However, the time delays will be explored in this study because time delay is a crucial parameter for control system design. Neglecting of time delays often introduces instability to the controlled closed loop. In this study, the triaxial accelerometers are employed to synchronize body movements with respiratory related signals, which ensures reliable estimation of time delays. 


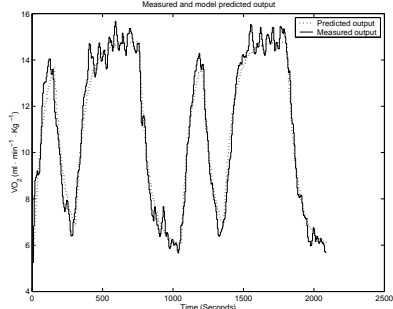

Fig. 4. Model estimation results

Based on the averaged data of PRBS input experiments, the time delay is determined by using Matlab Identification Toolbox. Two popular model selections criteria, Minimum Description Length (MDL) and Akaike Information Criterion (AIC), select the linear model as follows:

$$
y(k)=0.9308 y(k-1)+0.0692 u(k-1)+e(k),
$$

with sampling period $T_{s}=5$ seconds. The noise variance of model (7) is $3.5 \times 10^{-4}$. By transferring model (7) to its corresponding continuous form (8)

$$
Y(s)=\frac{1}{69.735 s+1} U(s),
$$

we can see that the dynamic part is a first order stable system (with time constant $T=69.735$ seconds and unit steady state gain) without time delay. This supports the belief [5] [30] [31] that the exponential rise in oxygen uptake directly reflected the rate of rise and drop in leg muscle oxygen consumption $\left(\dot{Q} O_{2}\right)$ at the onset and offset of exercises. The model fitting result is shown in Figure 4. Time constant and noise variance of model for each subject are summarized in Table III.

\begin{tabular}{ccccccc}
\hline Subjects & 1 & 2 & 3 & 4 & 5 & 6 \\
\hline $\begin{array}{c}\text { Time constant } \\
\text { (Seconds) }\end{array}$ & 45.4 & 61.8 & 69.3 & 63.3 & 65.8 & 64.1 \\
\hline $\begin{array}{c}\text { Noise variance } \\
\text { of models }\end{array}$ & 0.0013 & 0.0018 & 0.002 & 0.0017 & 0.0015 & 0.002 \\
\hline
\end{tabular}

TABLE III

TIME CONSTANT AND NOISE VARIANCE OF MODELS

Although the fitness of subjects is only determined based on our experiences rather than rigors assessment, a conjecture is proposed based on the calculated time constant (see Table III): fit subjects (subject 1) may have fast response (smaller time constants) with increasing or decreasing of workload.

\section{CONCLUSION}

This paper provides a Hammerstein model identification method to estimate oxygen uptake for treadmill walking exercises. The identification of dynamic linear component is decoupled by using a PRBS signal. In order to obtain good modeling results, breath by breath analysis is performed for PRBS input dynamical experiments. It is found that the time constants of identified first order ARX model for increasing and decreasing workload are identical within aerobic range.
This is an advantage for the controller design of oxygen uptake regulation for moderate treadmill exercises. By using the outputs of triaxial accelerometers to synchronize body movements with oxygen uptake, time delays of models are investigated. Our results support the opinion that time delay of the oxygen uptake dynamic can be ignored. Oxygen uptake of steady state experiments are calculated by using mixing chamber based respiratory measurement system. A RBF kernel SVM model is achieved based on the reliable steady state data, which is much better than traditional linear regression results. We believe that the proposed model may be useful in the prediction and regulation of oxygen uptake during treadmill exercises.

\section{REFERENCES}

[1] B. Franklin, ACSM's Guidelines for Exercise Testing and Prescription. New York: Lippincott Williams \& Wilkins, 2000.

[2] D. Dill, "Oxygen used in horizontal and grade walking and running on the treadmill," J Appl Physiol, pp. 19-22, 1965.

[3] W. T Van der Walt and C. Wyndham, "An equation for prediction of energy expenditure of walking and running," J Appl Physiol, pp. $559-563,1973$.

[4] K. Y. D. Abe and S. Niihata, "Effects of load carriage, load position, and walking speed on energy cost of walking," Applied Ergonomics, pp. 329-335, 2004.

[5] A. Hill and H. Lupton, "Muscular exercise, lactic acid and the supply and utilization of oxygen," Q. J. Med., pp. 135-171, 1923.

[6] D. Pearce and H. Milhorn, Jr., "Dynamic and steady-state respiratory responses to bicycle exercise," J. Appl. Physiol., no. 6, pp. 959-967, 1977.

[7] B. Whipp, S. Ward, N. Lamarra, J. Davis, and K. Wasserman, "Parameters of ventilatory and gas exchange dynamics during exercise,' J. Appl. Physiol., no. 6, pp. 1506-1513, 1982.

[8] U. Hoffmann, D. Efeld, H.-G. Wunderlich, and J. Stegemann, "Dynamic linearity of $v_{2}$ responses during aerobic exercise," European Journal of Applied Physiology, no. 2, pp. 139-144, 1992.

[9] D. Efeld, U. Hoffmann, and J. Stegemann, " $v o_{2}$ kinetics in subjects differing in aerobic capacity: investigation by spectral analysis," $E u$ ropean Journal of Applied Physiology, no. 5, pp. 508-515, 1987.

[10] E. Eskinat, S. Johnson, and W. Luyben, "Use of Hammerstein models in identification of nonlinear systems," AIChE J., vol. 37, pp. 255-268, 1991.

[11] S. Su, J. Bao, and P. Lee, "Control of multivariable hammerstein systems by using feedforward passivation," Ind. \& Eng. Chem. Res., no. 4, pp. 891-899, 2005.

[12] J. Allin and G. Inbar, "Fns parameter selection and upper limb characterization," IEEE Trans. Biomed. Eng., pp. 809-817, 1986.

[13] K. Hunt, M. Munih, N. Donaldson, and F. Barr, "Investigation of the hammerstein hypothesis in the modeling of electrically stimulated muscle," IEEE Trans. Biomed. Eng., no. 8, pp. 998-1009, 1998.

[14] L. Bernotas, P. Crago, and H. Chizeck, "A discrete-time model of electrically stimulated muscle," IEEE Trans. Biomed. Eng., pp. 829838, 1986.

[15] G. Maksym, R. Kearney, and J. Bates, "Nonparametric blockstructured modeling of lung tissue strip mechanics," Ann Biomed Eng, no. $2,1998$.

[16] I. Goethals., K. Pelckmans, J. Suykens, and B. De Moor, "Identification of mimo Hammerstein models using least squares support vector machines," Automatica, vol. 41, pp. 1263-1272, 2005.

[17] P. Falugi, L. Giarre, and G. Zappa, "Approximation of the feasible parameter set in worst-case identification of Hammerstein models," Automatica, vol. 41, pp. 1017-1024, 2005.

[18] H. Drucker, C. Burges, L.Kaufman, A. Smola, and V. Vapnik, "Support vector regression machines," in Advances in Neural Information Procession Systems, M. Mozer, M. Jordan, and T. Petsche, Eds. Cambridge, MA, 1997, pp. 155-161.

[19] E. Bai, "Decoupling the linear and nonlinear parts in hammerstein model identification," Automatica, vol. 40, pp. 671-676, 2004.

[20] — , "An optimal two-stage identification algorithm for hammersteinwiener nonlinear systems," Automatica, no. 3, pp. 333-338, 1998. 
[21] B. Ninness and S. Gibson, "Quantifying the accuracy of hammerstein model estimation," Automatica, vol. 38, pp. 2037-2051, 2002.

[22] N. Cristianini and J. Shawe-Taylor, An Introduction to Support Vector Machines and other kernel-based learning methods. UK: Cambridge University Press, 2000.

[23] A. Smola and B. Schlkopf, "A tutorial on support vector regression," Statistics and Computing, pp. 199-222, 2004.

[24] M. Espinoza, K. Pelckmans, L. Hoegaerts, J. Suykens, J. De Brabanter, and B. De Moor, "A comparative study of ls-svms applied to the silver box identi.cation problem," Symposium on nonlinear control systems NOLCOS, pp. 513-518, 2004.

[25] J. Suykens, Van Gestel, J. De Brabanter, B. De Moor, and J. Vandewalle, Least squares support vector machines. Singapore: WorldScientific, 2002.

[26] — , "Least squares support vector machines," Neurocomputing, vol. 48, pp. 85-105, 2002.

[27] L. Wang, S. Su, B. Celler, and A. Savkin, "Modeling of a gas concentration measurement system," in Proceedings of the 27th Annual International Conference of the IEEE Engineering in Medicine and Biology Society (EMBS), September, 2005, Shanghai, China.

[28] V. Vapnik, Statistical Learning Theory. New York: John Wiley, 1998.

[29] B. Schlkopf and A. Smola, Learning with kernels. Cambridge: MA: MIT Pres, 2002.

[30] DI Prampero, P. Davies, P. Cerretelli, and R.Margria, "An analysis of $\mathrm{O}_{2}$ debt contracted in submaximal exercise," J. Appl. Physiol., pp. 547-551, 1970.

[31] J. Hagberg, R. Hickson, A. Ehsani, and J. Holloszy, "Faster adjustment to and recovery from submaximal exercise in the trained state," J. Appl. Physiol., pp. 218-224, 1980. 